Research Article

\title{
Results of Positive Solutions for the Fractional Differential System on an Infinite Interval
}

\author{
Ying Wang $(\mathbb{D}$, Jun Yang, and Yumei Zi \\ School of Mathematics and Statistics, Linyi University, Linyi 276000, Shandong, China \\ Correspondence should be addressed to Ying Wang; lywy1981@163.com
}

Received 6 February 2020; Accepted 28 February 2020; Published 5 May 2020

Guest Editor: Chuanjun Chen; cjchen@ytu.edu.cn

Copyright (C) 2020 Ying Wang et al. This is an open access article distributed under the Creative Commons Attribution License, which permits unrestricted use, distribution, and reproduction in any medium, provided the original work is properly cited.

The chief topic of this paper is to investigate the fractional differential system on an infinite interval. By introducing an appropriate compactness criterion in a special function space and applying the Schauder fixed-point theorem and the Banach contraction mapping principle, we established the results for the existence and uniqueness of positive solutions. An example is then given to show the utilization of the main results.

\section{Introduction}

In this paper, we investigate the following fractional differential system on an infinite interval:

$$
\begin{cases}D_{0^{+}}^{\alpha_{1}} u(t)+f_{1}(t, u(t), v(t))=0, & \\ D_{0^{+}}^{\alpha_{2}} v(t)+f_{2}(t, u(t), v(t))=0, & 0<t<+\infty, \\ u(0)=u^{\prime}(0)=u^{\prime \prime}(0)=\cdots=u^{(n-2)}(0)=0, & D_{0^{+}}^{\alpha_{1}-1} u(+\infty)=\mu_{1} \int_{0}^{\infty} a_{1}(t) u(t) \mathrm{d} A_{1}(t), \\ v(0)=v^{\prime}(0)=v^{\prime \prime}(0)=\cdots=v^{(m-2)}(0)=0, & D_{0^{+}}^{\alpha_{2}-1} v(+\infty)=\mu_{2} \int_{0}^{\infty} a_{2}(t) v(t) \mathrm{d} A_{2}(t),\end{cases}
$$

where $1 \leq n-1<\alpha_{1} \leq n, 1 \leq m-1<\alpha_{2} \leq m$, and $n, m \geq 2$, $D_{0^{+}}^{\alpha_{i}}$ is the Riemann-Liouville derivative operator. $\mu_{i}>0$ is a constant, $\int_{0}^{\infty} a_{1}(t) u(t) \mathrm{d} A_{1}(t)$ and $\int_{0}^{\infty} a_{2}(t) v(t) \mathrm{d} A_{2}(t)$ denote the Riemann-Stieltjes integral, and $A_{i}$ is a function of bounded positive variation. $a_{i} \in L[0,+\infty), \quad \mu_{i} \int_{0}^{\infty}$ $a_{i}(t) t^{\alpha_{i}-1} \mathrm{~d} A_{i}(t)<\Gamma\left(\alpha_{i}\right), \int_{0}^{\infty} a_{i}(t) \mathrm{d} A_{i}(t)<+\infty$, and $f_{i}:[0$, $+\infty) \times[0,+\infty) \times[0,+\infty) \longrightarrow[0,+\infty)$ is continuous, $i=1$, 2.

Numerous models in physics, chemistry, biology, medicine, and other fields have promoted the research of differential equations, for instance, evaluation of water quality on receiving water [1], and the advection-dispersion equation can be formulated as shown for the case of one-dimensional flow:

$$
\frac{\partial C}{\partial t}+u \frac{\partial C}{\partial x}=D_{L} \frac{\partial^{2} C}{\partial t^{2}}-f(C),
$$

where $C$ is the concentration of a generic pollutant, $t$ is the time, $x$ is the longitudinal displacement, $u$ is the velocity, $D_{L}$ is the diffusion coefficient, and $f(C)$ is a generic term for reactions involving the pollutant $C$. Westerlund [2] established a one-dimensional model to describe the transmission of the electromagnetic wave: 


$$
\mu \varepsilon \frac{\partial^{2} E(x, t)}{\partial x^{2}}+\mu \varepsilon \zeta D_{t}^{v} E(x, t)+\frac{\partial^{2} E(x, t)}{\partial t^{2}}=0,
$$

where $\mu, \varepsilon$, and $\zeta$ are constants and $D_{t}^{\nu} E(x, t)=$ $\left(\partial^{\nu} E(x, t)\right) / \partial t^{\nu}$ is a fractional derivative. In the process of establishing the model, $k$-Hessian equations [3], Sobolev equations [4], and Schrödinger elliptic equations [5, 6], there are also huge applications.

Under the proper initial or boundary conditions, to study the positive solution of the above models is very necessary; especially, for the boundary value problems on the infinite interval, many authors put their interest in it [7-16]. Liang and Zhang [17] applied the fixed-point theorem to obtain the existence of positive solutions for the following fractional differential equation:

$$
\begin{cases}D_{0^{+}}^{\alpha} u(t)+a(t) f(u(t))=0, & 0<t<+\infty, \\ u(0)=u^{\prime}(0)=0, & \lim _{t \rightarrow+\infty} D_{0^{+}}^{\alpha-1} u(t)=\sum_{i=1}^{m-2} \beta_{i} u\left(\xi_{i}\right),\end{cases}
$$

where $2<\alpha \leq 3, D_{0^{+}}^{\alpha}$ is the Riemann-Liouville fractional derivative, $0<\xi_{1}<\xi_{2}<\cdots<\xi_{m-2}<+\infty, \beta_{i}>0,0<\sum_{i=1}^{m-2} \beta_{i} u$ $\left(\xi_{i}\right)<\Gamma(\alpha)$, and $f:[0,+\infty) \longrightarrow[0,+\infty)$ is continuous.

For all we know, there are few studies on fractional differential systems of infinite intervals, although it is necessary to do so. In this paper, we aimed at getting the existence and uniqueness of positive solutions for system (1) on infinite interval. Compared with the existing literature, the innovations of this paper are as follows. Firstly, the paper which we discuss is the system rather than a single equation. Secondly, we study the system with integral boundary value conditions on infinite intervals, which are more general than those of two-point, three-point, and multipoint boundary value condition. At last, we use two different techniques: the Schauder fixed-point theorem and the Banach contraction mapping principle, for system (1), not only to obtain the existence of positive solutions but also the uniqueness of positive solutions.

\section{Preliminaries and Lemmas}

Definition 1 (see $[18,19]$ ). Let $\alpha>0$ and $u$ be piecewise continuous on $(0,+\infty)$ and integrable on any finite subinterval of $[0,+\infty)$. Then, for $t>0$, we call

$$
I_{0^{+}}^{\alpha} u(t)=\frac{1}{\Gamma(\alpha)} \int_{0}^{t}(t-s)^{\alpha-1} u(s) \mathrm{d} s
$$

the Riemann-Liouville fractional integral of $u$ of order $\alpha$.

Definition 2 (see $[18,19])$. The Riemann-Liouville fractional derivative of order $\alpha>0, n-1 \leq \alpha<n, n \in \mathbb{N}$, is defined as

$$
D_{0^{+}}^{\alpha} u(t)=\frac{1}{\Gamma(n-\alpha)}\left(\frac{\mathrm{d}}{\mathrm{d} t}\right)^{n} \int_{0}^{t}(t-s)^{n-\alpha-1} u(s) \mathrm{d} s,
$$

where $\mathbb{N}$ denotes the natural number set and the function $u(t)$ is $n$ times continuously differentiable on $[0,+\infty)$.

Lemma 1 (see $[18,19])$. Let $\alpha>0$, and if the fractional derivative $D_{0^{+}}^{\alpha-1} u(t)$ and $D_{0^{+}}^{\alpha} u(t)$ are continuous on $[0,+\infty)$, then,

$$
I_{0^{+}}^{\alpha} D_{0^{+}}^{\alpha} u(t)=u(t)+c_{1} t^{\alpha-1}+c_{2} t^{\alpha-2}+\cdots+c_{n} t^{\alpha-n}
$$

where $c_{1}, c_{2}, \ldots, c_{n} \in(-\infty,+\infty), n$ is the smallest integer greater than or equal to $\alpha$.

Lemma 2. Let $y_{i} \in C(0,+\infty) \cap L[0,+\infty)$; then, the fractional system

$$
\left\{\begin{array}{l}
D_{0+}^{\alpha_{1}} u(t)+y_{1}(t)=0 \\
D_{0+}^{\alpha_{1}} v(t)+y_{2}(t)=0 \\
0<t<+\infty \\
u(0)=u^{\prime}(0)=u^{\prime \prime}(0)=\cdots=u^{(n-2)}(0)=0 \\
D_{0+}^{\alpha_{1}-1} u(+\infty)=\mu_{1} \int_{0}^{\infty} a_{1}(t) u(t) \mathrm{d} A_{1}(t) \\
v(0)=v^{\prime}(0)=v^{\prime \prime}(0)=\cdots=v^{(m-2)}(0)=0 \\
D_{0+}^{\alpha_{2}-1} v(+\infty)=\mu_{2} \int_{0}^{\infty} a_{2}(t) v(t) \mathrm{d} A_{2}(t)
\end{array}\right.
$$

has a unique integral representation

$$
\left\{\begin{array}{l}
u(t)=\int_{0}^{\infty} G_{1}(t, s) y_{1}(s) \mathrm{d} s \\
v(t)=\int_{0}^{\infty} G_{2}(t, s) y_{2}(s) \mathrm{d} s
\end{array}\right.
$$

where

$$
\begin{aligned}
& G_{i}(t, s)=G_{i 1}=(t, s)+G_{i 2}(t, s), \quad i=1,2, \\
& G_{i 1}(t, s)=\frac{1}{\Gamma\left(\alpha_{i}\right)} \begin{cases}t^{\alpha-1}-(t-s)^{\alpha_{i}-1}, & 0 \leq s \leq t \leq+\infty, \\
t^{\alpha_{i}-1}, & 0 \leq t \leq s \leq+\infty,\end{cases} \\
& G_{i 2}(t, s)=\frac{\mu_{i} t^{\alpha_{i}-1}}{\Gamma\left(\alpha_{i}\right)-\mu_{i} \int_{0}^{\infty} a_{i}(t) t^{\alpha_{i}-1} \mathrm{~d} A_{i}(t)} \int_{0}^{\infty} a_{i}(t) G_{i 1}(t, s) \mathrm{d} A_{i}(t) .
\end{aligned}
$$


Proof. By Lemma 1, the equations in system (8) can be transformed into the equivalent integral equations

$$
\begin{aligned}
& u(t)=-I_{0+}^{\alpha_{1}} y_{1}(t)+c_{1} t^{\alpha_{1}-1}+c_{2} t^{\alpha_{1}-2}+\cdots+c_{n} t^{\alpha_{1}-n}, c_{j} \in(-\infty,+\infty), \quad j=1,2, \ldots, n, \\
& v(t)=-I_{0+}^{\alpha_{2}} y_{2}(t)+\bar{c}_{1} t^{\alpha_{2}-1}+\bar{c}_{2} t^{\alpha_{2}-2}+\cdots+c_{m} t^{\alpha_{2}-m}, \bar{c}_{j} \in(-\infty,+\infty), \quad \bar{j}=1,2, \ldots, m,
\end{aligned}
$$

that is,

$$
\begin{aligned}
& u(t)=-\frac{1}{\Gamma\left(\alpha_{1}\right)} \int_{0}^{t}(t-s)^{\alpha_{1}-1} y_{1}(s) \mathrm{d} s+c_{1} t^{\alpha_{1}-1}+c_{2} t^{\alpha_{1}-2}+\cdots+c_{n} t^{\alpha_{1}-n}, c_{j} \in(-\infty,+\infty), \quad j=1,2, \ldots, n, \\
& v(t)=-\frac{1}{\Gamma\left(\alpha_{2}\right)} \int_{0}^{t}(t-s)^{\alpha_{2}-1} y_{2}(s) \mathrm{d} s+\bar{c}_{1} t^{\alpha_{2}-1}+\bar{c}_{2} t^{\alpha_{2}-2}+\cdots+\bar{c}_{m} t^{\alpha_{2}-m}, \bar{c}_{j} \in(-\infty,+\infty), \quad j=1,2, \ldots, m
\end{aligned}
$$

Since

$$
\begin{aligned}
& u(0)=u^{\prime}(0)=\cdots=u^{(n-2)}=0, \\
& v(0)=v^{\prime}(0)=\cdots=v^{(m-2)}(0)=0,
\end{aligned}
$$

we have

$$
\begin{aligned}
& c_{2}=c_{3}=\cdots=c_{n}=0, \\
& \bar{c}_{2}=\bar{c}_{3}=\cdots=\bar{c}_{m}=0 .
\end{aligned}
$$

So,

$$
\begin{aligned}
& u(t)=-\frac{1}{\Gamma\left(\alpha_{1}\right)} \int_{0}^{t}(t-s)^{\alpha_{1}-1} y_{1}(s) \mathrm{d} s+c_{1} t^{\alpha_{1}-1}, \\
& v(t)=-\frac{1}{\Gamma\left(\alpha_{2}\right)} \int_{0}^{t}(t-s)^{\alpha_{2}-1} y_{2}(s) \mathrm{d} s+\bar{c}_{1} t^{\alpha_{2}-1} .
\end{aligned}
$$

We also have

$$
\begin{aligned}
& D_{0^{+}}^{\alpha_{1}-1} u(t)=c_{1} \Gamma\left(\alpha_{1}\right)-\int_{0}^{t} y_{1}(s) \mathrm{d} s, \\
& D_{0^{+}}^{\alpha_{2}-1} v(t)=\bar{c}_{1} \Gamma\left(\alpha_{2}\right)-\int_{0}^{t} y_{2}(s) \mathrm{d} s .
\end{aligned}
$$

For

$$
\begin{aligned}
& D_{0^{+}}^{\alpha_{1}-1} u(+\infty)=\mu_{1} \int_{0}^{\infty} a_{1}(s) u(s) \mathrm{d} A_{1}(s), \\
& D_{0^{+}}^{\alpha_{2}-1} v(+\infty)=\mu_{2} \int_{0}^{\infty} a_{2}(s) v(s) \mathrm{d} A_{2}(s),
\end{aligned}
$$

we obtain

$$
\begin{aligned}
& c_{1}=\frac{1}{\Gamma\left(\alpha_{1}\right)}\left(\mu_{1} \int_{0}^{\infty} a_{1}(s) u(s) \mathrm{d} A_{1}(s)+\int_{0}^{+\infty} y_{1}(s) \mathrm{d} s\right), \\
& \bar{c}_{1}=\frac{1}{\Gamma\left(\alpha_{2}\right)}\left(\mu_{2} \int_{0}^{\infty} a_{2}(s) v(s) \mathrm{d} A_{2}(s)+\int_{0}^{+\infty} y_{2}(s) \mathrm{d} s\right) .
\end{aligned}
$$

Combining (15) and (18), we have

$$
\begin{aligned}
u(t) & =-\frac{1}{\Gamma\left(\alpha_{1}\right)} \int_{0}^{t}(t-s)^{\alpha_{1}-1} y_{1}(s) \mathrm{d} s+c_{1} t^{\alpha_{1}-1} \\
& =\int_{0}^{\infty} G_{11}(t, s) y_{1}(s) \mathrm{d} s+\frac{\mu_{1} t^{\alpha_{1}-1}}{\Gamma\left(\alpha_{1}\right)} \int_{0}^{\infty} a_{1}(t) u(t) \mathrm{d} A_{1}(t),
\end{aligned}
$$

$$
\begin{aligned}
v(t) & =-\frac{1}{\Gamma\left(\alpha_{2}\right)} \int_{0}^{t}(t-s)^{\alpha_{2}-1} y_{2}(s) \mathrm{d} s+\bar{c}_{1} t^{\alpha_{2}-1} \\
& =\int_{0}^{\infty} G_{21}(t, s) y_{2}(s) \mathrm{d} s+\frac{\mu_{2} t^{\alpha_{2}-1}}{\Gamma\left(\alpha_{2}\right)} \int_{0}^{\infty} a_{2}(t) v(t) \mathrm{d} A_{2}(t) .
\end{aligned}
$$

(19) and (20) are multiplied by $a_{1}(t)$ and $a_{2}(t)$, respectively, and then solved the integral from 0 to $+\infty$ with respect to $A_{1}(t)$ and $A_{2}(t)$; then, we have

$$
\begin{aligned}
& \int_{0}^{\infty} a_{1}(t) u(t) \mathrm{d} A_{1}(t)=\frac{\Gamma\left(\alpha_{1}\right)}{\Gamma\left(\alpha_{1}\right)-\mu_{1} \int_{0}^{\infty} a_{1}(t) \mathrm{d} A_{1}(t)} \int_{0}^{\infty} a_{1}(t) \int_{0}^{\infty} G_{11}(t, s) y_{1}(s) \mathrm{d} s \mathrm{~d} A_{1}(t), \\
& \int_{0}^{\infty} a_{2}(t) v(t) \mathrm{d} A_{2}(t)=\frac{\Gamma\left(\alpha_{2}\right)}{\Gamma\left(\alpha_{2}\right)-\mu_{2} \int_{0}^{\infty} a_{2}(t) \mathrm{d} A_{2}(t)} \int_{0}^{\infty} a_{2}(t) \int_{0}^{\infty} G_{21}(t, s) y_{2}(s) \mathrm{d} s \mathrm{~d} A_{2}(t) .
\end{aligned}
$$


Therefore,

$$
\begin{aligned}
u(t)= & \int_{0}^{\infty} G_{11}(t, s) y_{1}(s) \mathrm{d} s+\frac{\mu_{1} t^{\alpha_{1}-1}}{\Gamma\left(\alpha_{1}\right)} \int_{0}^{\infty} a_{1}(t) u(t) \mathrm{d} A_{1}(t) \\
= & \int_{0}^{\infty} G_{11}(t, s) y_{1}(s) \mathrm{d} s+\frac{\mu_{1} t^{\alpha_{1}-1}}{\Gamma\left(\alpha_{1}\right)-\mu_{1} \int_{0}^{\infty} a_{1}(t) \mathrm{d} A_{1}(t)} \\
& \cdot \int_{0}^{\infty} a_{1}(t) \int_{0}^{\infty} G_{11}(t, s) y_{1}(s) \mathrm{d} s \mathrm{~d} A_{1}(t) \\
= & \int_{0}^{\infty} G_{11}(t, s) y_{1}(s) \mathrm{d} s+\int_{0}^{\infty} G_{12}(t, s) y_{1}(s) \mathrm{d} s, \\
v(t)= & \int_{0}^{\infty} G_{21}(t, s) y_{2}(s) \mathrm{d} s+\frac{\mu_{2} t^{\alpha_{2}-1}}{\Gamma\left(\alpha_{2}\right)} \int_{0}^{\infty} a_{2}(t) v(t) \mathrm{d} A_{2}(t) \\
= & \int_{0}^{\infty} G_{21}(t, s) y_{2}(s) \mathrm{d} s+\frac{\mu_{2} t^{\alpha_{2}-1}}{\Gamma\left(\alpha_{2}\right)-\mu_{2} \int_{0}^{\infty} a_{2}(t) \mathrm{d} A_{2}(t)} \\
& \cdot \int_{0}^{\infty} a_{2}(t) \int_{0}^{\infty} G_{21}(t, s) y_{2}(s) \mathrm{d} s \mathrm{~d} A_{2}(t) \\
= & \int_{0}^{\infty} G_{21}(t, s) y_{2}(s) \mathrm{d} s+\int_{0}^{\infty} G_{22}(t, s) y_{2}(s) \mathrm{d} s .
\end{aligned}
$$

So, (9) holds. The proof is completed.

Lemma 3. The Green function in Lemma 2 has the following properties:

(1) $G_{i 1}(t, s)$ is continuous and $G_{i 1}(t, s) \geq 0,(t, s) \in[0$, $+\infty) \times[0,+\infty)$.

(2) $\left(G_{i 1}(t, s) / 1+t^{\alpha_{i}-1}\right) \leq\left(1 / \Gamma\left(\alpha_{i}\right)\right),\left(G_{i}(t, s) / 1+t^{\alpha_{i}-1}\right)$ $\leq \omega,(t, s) \in[0,+\infty) \times[0,+\infty)$,

where

$$
\omega=\max \left\{\begin{array}{c}
\frac{1}{\Gamma\left(\alpha_{1}\right)}+\frac{\Gamma\left(\alpha_{1}\right)+\mu_{1} \int_{0}^{\infty} a_{1}(t) \mathrm{d} A_{1}(t)}{\Gamma\left(\alpha_{1}\right)\left(\Gamma\left(\alpha_{1}\right)-\mu_{1} \int_{0}^{\infty} a_{1}(t) t^{\alpha_{1}-1} \mathrm{~d} A_{1}(t)\right)}, \\
\frac{1}{\Gamma\left(\alpha_{2}\right)}+\frac{\Gamma\left(\alpha_{2}\right)+\mu_{2} \int_{0}^{\infty} a_{2}(t) \mathrm{d} A_{2}(t)}{\Gamma\left(\alpha_{2}\right)\left(\Gamma\left(\alpha_{2}\right)-\mu_{2} \int_{0}^{\infty} a_{2}(t) t^{\alpha_{2}-1} \mathrm{~d} A_{2}(t)\right)}
\end{array}\right\} .
$$

The space $X=E_{1} \times E_{2}$ will be used in the study of system (1), where

$$
\begin{aligned}
& E_{1}=\left\{u \in C[0,+\infty): \sup _{t \in[0,+\infty)} \frac{u(t)}{1+t^{\alpha_{1}-1}}<+\infty\right\}, \\
& E_{2}=\left\{v \in C[0,+\infty): \sup _{t \in[0,+\infty)} \frac{v(t)}{1+t^{\alpha_{2}-1}}<+\infty\right\} .
\end{aligned}
$$

Then, $\left(E_{1},\|\cdot\|\right)$ and $\left(E_{2},\|\cdot\|\right)$ are the Banach space with the norm

$$
\begin{gathered}
\|u\|=\sup _{t \in[0,+\infty)} \frac{u(t)}{1+t^{\alpha_{1}-1}} \\
\|v\|=\sup _{t \in[0,+\infty)} \frac{v(t)}{1+t^{\alpha_{2}-1}} .
\end{gathered}
$$

Clearly, $(X,\|\cdot\|)$ is a Banach space with the norm $\|u, v\|=\|u\|+\|v\|$. Define nonlinear integral operators $T_{i}$ : $X \longrightarrow E_{i}$ and $T: X \longrightarrow X$ by

$$
\begin{aligned}
T_{i}(u, v)(t) & =\int_{0}^{\infty} G_{i}(t, s) f_{i}(s, u(s), v(s)) \mathrm{d} s, \quad i=1,2, \\
T(u, v) & =\left(T_{1}(u, v), T_{2}(u, v)\right) .
\end{aligned}
$$

Thus, the existence of solutions to system (1) is equivalent to the existence of solutions in $X$ for operator equation $T(u, v)=(u, v)$ defined by (27).

Lemma 4 (see $[20,21]$ ). Let $E$ be defined as (24) and $M$ be any bounded subset of $E$. Then, $M$ is relatively compact in $E$ if $\left\{\left(x(t) / 1+t^{\alpha-1}\right): x \in M\right\}$ is equicontinuous on any finite subinterval of J, and for any given $\varepsilon>0$, there exists $N>0$ such that $\left|\left(x\left(t_{1}\right) / 1+t_{1}^{\alpha-1}\right)-\left(x\left(t_{2}\right) / 1+t_{2}^{\alpha-1}\right)\right|<\varepsilon$ uniformly with respect to all $x \in M$, and $t_{1}, t_{2}>N$.

\section{Main Results}

We list the conditions to be used later: $\left(\mathbf{H}_{1}\right)$ there exist nonnegative functions $p_{i}(t), g_{i}(t), h_{i}(t) \in L^{1}[0,+\infty)$ and $t^{\alpha_{1}-1} g_{i}(t), t^{\alpha_{2}-1} h_{i}(t) \in L^{1}[0,+\infty)$ such that

$$
\begin{aligned}
\left|f_{i}(t, u, v)\right| \leq & p_{i}(t)+g_{i}(t)|u|+h_{i}(t)|v|,(t, u, v) \in[0,+\infty) \\
& \times[0,+\infty) \times[0,+\infty) .
\end{aligned}
$$

$\left(\mathbf{H}_{2}\right)\left|f_{i}(t, 0,0)\right| \in L^{1}[0,+\infty)$, there exist nonnegative functions $g_{i}(t), \quad h_{i}(t) \in L^{1}[0, \quad+\infty)$ and $t^{\alpha_{1}-1} g_{i}(t)$, $t^{\alpha_{2}-1} h_{i}(t) \in L^{1}[0,+\infty)$ such that

$$
\begin{array}{r}
\left|f_{i}\left(t, u_{1}, v_{1}\right)-f_{i}\left(t, u_{2}, v_{2}\right)\right| \leq g_{i}(t)\left|u_{1}-u_{2}\right|+h_{i}(t)\left|v_{1}-v_{2}\right|, \\
\left(t, u_{1}, v_{1}\right),\left(t, u_{1}, v_{1}\right) \in[0,+\infty) \times[0,+\infty) \times[0,+\infty) .
\end{array}
$$

Remark 1. If $\left(\mathbf{H}_{1}\right)$ holds, then

$$
\int_{0}^{\infty}\left|f_{i}(t, u(t), v(t))\right| \mathrm{d} t \leq p_{i}^{*}+\left(g_{i}^{*}+h_{i}^{*}\right)\|(u, v)\|, \quad(u, v) \in X,
$$

where

$$
\begin{aligned}
& p_{i}^{*}=\int_{0}^{\infty} p_{i}(t) \mathrm{d} t \\
& g_{i}^{*}=\int_{0}^{\infty}\left(1+t^{\alpha_{1}-1}\right) g_{i}(t) \mathrm{d} t \\
& h_{i}^{*}=\int_{0}^{\infty}\left(1+t^{\alpha_{2}-1}\right) h_{i}(t) \mathrm{d} t
\end{aligned}
$$


In fact, by $\left(\mathbf{H}_{1}\right)$, for any $(u, v) \in X$, we have

$$
\begin{aligned}
& \int_{0}^{\infty}\left|f_{i}(t, u(t), v(t))\right| \mathrm{d} t \\
& \quad \leq \int_{0}^{\infty}\left(p_{i}(t)+g_{i}(t)|u(t)|+h_{i}(t)|v(t)|\right) \mathrm{d} t \\
& \quad=\int_{0}^{\infty}\left(p_{i}(t)+\left(1+t^{\alpha_{1}-1}\right) g_{i}(t) \frac{|u(t)|}{1+t^{\alpha_{1}-1}}+\left(1+t^{\alpha_{2}-1}\right) h_{i}(t) \frac{|v(t)|}{1+t^{\alpha_{2}-1}}\right) \mathrm{d} t \\
& \quad \leq \int_{0}^{\infty}\left(p_{i}(t)+\left(1+t^{\alpha_{1}-1}\right) g_{i}(t)\|u\|+\left(1+t^{\alpha_{2}-1}\right) h_{i}(t)\|v\|\right) \mathrm{d} t \\
& \quad=\int_{0}^{\infty} p_{i}(t) \mathrm{d} t+\int_{0}^{\infty}\left(1+t^{\alpha_{1}-1}\right) g_{i}(t) \mathrm{d} t\|u\|+\int_{0}^{\infty}\left(1+t^{\alpha_{2}-1}\right) h_{i}(t) \mathrm{d} t\|v\| \\
& \quad=p_{i}^{*}+g_{i}^{*}\|u\|+h_{i}^{*}\|v\| \leq p_{i}^{*}+\left(g_{i}^{*}+h_{i}^{*}\right)\|(u, v)\| .
\end{aligned}
$$

Theorem 1. Assume that $\left(\boldsymbol{H}_{1}\right)$ holds; then, $T: X \longrightarrow X$ is a completely continuous operator.
Proof. First, we show that $T: X \longrightarrow X$ is continuous. Suppose $\left\{\left(u_{n}, v_{n}\right)\right\} \subset X,(u, v) \in X$ with $\left\|\left(u_{n}, v_{n}\right)-(u, v)\right\| \longrightarrow 0$ $(n \longrightarrow+\infty)$, and there exists a constant $r>0$ such that $\left\|\left(u_{n}, v_{n}\right)\right\| \leq r$ and $\|(u, v)\| \leq r$. By $\left(\mathbf{H}_{1}\right)$ and (30), we have

$$
\begin{aligned}
& \left|\int_{0}^{\infty} G_{1}(t, s) f_{1}\left(s, u_{n}(s), v_{n}(s)\right) \mathrm{d} s-\int_{0}^{\infty} G_{1}(t, s) f_{1}(s, u(s), v(s)) \mathrm{d} s\right| \\
& \quad \leq \int_{0}^{\infty} G_{1}(t, s) f_{1}\left(s, u_{n}(s), v_{n}(s)\right) \mathrm{d} s+\int_{0}^{\infty} G_{1}(t, s) f_{1}(s, u(s), v(s)) \mathrm{d} s \\
& \quad \leq \omega \int_{0}^{\infty} f_{1}\left(s, u_{n}(s), v_{n}(s)\right) \mathrm{d} s+\omega \int_{0}^{\infty} f_{1}(s, u(s), v(s)) \mathrm{d} s \\
& \quad \leq 2 \omega\left(p_{1}^{*}+g_{1}^{*}\|u\|+h_{1}^{*}\|v\|\right) \leq 2 \omega\left(p_{1}^{*}+\left(g_{1}^{*}+h_{1}^{*}\right)\|(u, v)\|\right)<+\infty
\end{aligned}
$$

From $\left(\mathbf{H}_{1}\right)$ and (33), for any $\varepsilon>0$, there exists sufficiently large $M_{0}$ such that

$$
\begin{aligned}
& \int_{M_{0}}^{\infty} G_{1}(t, s) f_{1}\left(s, u_{n}(s), v_{n}(s)\right) \mathrm{d} s+\int_{M_{0}}^{\infty} G_{1}(t, s) f_{1} \\
& \cdot(s, u(s), v(s)) \mathrm{d} s<\frac{\varepsilon}{2} .
\end{aligned}
$$

On the contrary, by the continuity of $f_{1}(t, u, v)$ on $\left[0, M_{0}\right] \times\left[0,\left(1+M_{0}^{\alpha_{1}-1}\right) r\right] \times\left[0,\left(1+M_{0}^{\alpha_{1}-1}\right) r\right]$, there exists $N>0$ such that when $n>N$ and $t \in\left[0, M_{0}\right]$, we have

$$
\left|f_{1}\left(s, u_{n}(s), v_{n}(s)\right)-f_{1}(s, u(s), v(s))\right|<\frac{\varepsilon}{2 \omega M_{0}} .
$$

Hence, for any $t \in[0,+\infty)$ and $n>N$, we obtain

$$
\begin{aligned}
& \left|\frac{T_{1}\left(u_{n}, v_{n}\right)(t)}{1+t^{\alpha_{1}-1}}-\frac{T_{1}(u, v)(t)}{1+t^{\alpha_{1}-1}}\right| \\
& =\left|\int_{0}^{\infty} \frac{G_{1}(t, s)}{1+t^{\alpha_{1}-1}} f_{1}\left(s, u_{n}(s), v_{n}(s)\right) \mathrm{d} s-\int_{0}^{\infty} \frac{G_{1}(t, s)}{1+t^{\alpha_{1}-1}} f_{1}(s, u(s), v(s)) \mathrm{d} s\right| \\
& \leq \int_{0}^{M_{0}} \frac{G_{1}(t, s)}{1+t^{\alpha_{1}-1}}\left|f_{1}\left(s, u_{n}(s), v_{n}(s)\right)-f_{1}(s, u(s), v(s))\right| \mathrm{d} s \\
& \quad+\int_{M_{0}}^{\infty} \frac{G_{1}(t, s)}{1+t^{\alpha_{1}-1}} f_{1}\left(s, u_{n}(s), v_{n}(s)\right) \mathrm{d} s+\int_{M_{0}}^{\infty} \frac{G_{1}(t, s)}{1+t^{\alpha_{1}-1}} f_{1}(s, u(s), v(s)) \mathrm{d} s
\end{aligned}
$$




$$
\begin{aligned}
\leq & \int_{0}^{M_{0}} \frac{G_{1}(t, s)}{1+t^{\alpha_{1}-1}}\left|f_{1}\left(s, u_{n}(s), v_{n}(s)\right)-f_{1}(s, u(s), v(s))\right| \mathrm{d} s \\
& +\int_{M_{0}}^{\infty} G_{1}(t, s) f_{1}\left(s, u_{n}(s), v_{n}(s)\right) \mathrm{d} s+\int_{M_{0}}^{\infty} G_{1}(t, s) f_{1}(s, u(s), v(s)) \mathrm{d} s \\
< & \omega \int_{0}^{M_{0}}\left|f_{1}\left(s, u_{n}(s), v_{n}(s)\right) \mathrm{d} s-f_{1}(s, u(s), v(s))\right| \mathrm{d} s+\frac{\varepsilon}{2} \\
< & \frac{\varepsilon}{2}+\frac{\varepsilon}{2}=\varepsilon .
\end{aligned}
$$

Thus, we know that $\left\|T_{1}\left(u_{n}, v_{n}\right)-T_{1}(u, v)\right\| \longrightarrow 0$ $(n \longrightarrow+\infty)$. By the similar proof as (33)-(36), we know $\left\|T_{2}\left(u_{n}, v_{n}\right)-T_{2}(u, v)\right\| \longrightarrow 0(n \longrightarrow+\infty)$. So, $T: X \longrightarrow X$ is continuous.

Next, we show that $T: X \longrightarrow X$ is relatively compact. Let $\Omega$ be a bounded subset of $X$; then, there exists constant $M>0$ such that $\|(u, v)\| \leq M,(u, v) \in \Omega$. For any $(u, v) \in \Omega, t \in[0$, $+\infty)$, and by (32), we obtain

$$
\begin{aligned}
\left|\frac{T_{1}\left(u_{n}, v_{n}\right)(t)}{1+t^{\alpha_{1}-1}}\right| & =\left|\int_{0}^{\infty} \frac{G_{1}(t, s)}{1+t^{\alpha_{1}-1}} f_{1}\left(s, u_{n}(s), v_{n}(s)\right) \mathrm{d} s\right| \\
& \leq \omega \int_{0}^{\infty}\left|f_{1}\left(s, u_{n}(s), v_{n}(s)\right)\right| \mathrm{d} s \\
& \leq \omega\left(p_{1}^{*}+\left(g_{1}^{*}+h_{1}^{*}\right)\|(u, v)\|\right) \\
& \leq \omega\left(p_{1}^{*}+\left(g_{1}^{*}+h_{1}^{*}\right) M\right)<+\infty .
\end{aligned}
$$

Similarly, we have

$$
\begin{aligned}
\left|\frac{T_{2}\left(u_{n}, v_{n}\right)(t)}{1+t^{\alpha_{2}-1}}\right| & \leq \omega\left(p_{1}^{*}+\left(g_{1}^{*}+h_{1}^{*}\right)\|(u, v)\|\right) \\
& \leq \omega\left(p_{1}^{*}+\left(g_{1}^{*}+h_{1}^{*}\right) M\right)<+\infty .
\end{aligned}
$$

Consequently, $T(\Omega)$ is uniformly bounded.

Given $I \subset[0,+\infty)$ be any compact interval. For any $t_{1}$, $t_{2} \in I, t_{1}<t_{2}$ and $(u, v) \in \Omega$, we deduce

$$
\begin{aligned}
& \left|\frac{T_{1}(u, v)\left(t_{2}\right)}{1+t_{2}^{\alpha_{1}-1}}-\frac{T_{1}(u, v)\left(t_{1}\right)}{1+t_{1}^{\alpha_{1}-1}}\right| \\
& =\left|\int_{0}^{\infty} \frac{G_{1}\left(t_{2}, s\right)}{1+t_{2}^{\alpha_{1}-1}} f_{1}(s, u(s), v(s)) \mathrm{d} s-\int_{0}^{\infty} \frac{G_{1}\left(t_{1}, s\right)}{1+t_{1}^{\alpha_{1}-1}} f_{1}(s, u(s), v(s)) \mathrm{d} s\right| \\
& \leq \int_{0}^{\infty}\left|\frac{G_{11}\left(t_{2}, s\right)}{1+t_{2}^{\alpha_{1}-1}}-\frac{G_{11}\left(t_{1}, s\right)}{1+t_{1}^{\alpha_{1}-1}}\right| f_{1}(s, u(s), v(s)) \mathrm{d} s \\
& +\int_{0}^{\infty}\left|\frac{G_{12}\left(t_{2}, s\right)}{1+t_{2}^{\alpha_{1}-1}}-\frac{G_{12}\left(t_{1}, s\right)}{1+t_{1}^{\alpha_{1}-1}}\right| f_{1}(s, u(s), v(s)) \mathrm{d} s \\
& \leq \int_{0}^{\infty}\left|\frac{G_{11}\left(t_{2}, s\right)}{1+t_{2}^{\alpha_{1}-1}}-\frac{G_{11}\left(t_{1}, s\right)}{1+t_{1}^{\alpha_{1}-1}}\right| f_{1}(s, u(s), v(s)) \mathrm{d} s \\
& +\left|\frac{t_{2}^{\alpha_{1}-1}}{1+t_{2}^{\alpha_{1}-1}}-\frac{t_{1}^{\alpha_{1}-1}}{1+t_{1}^{\alpha_{1}-1}}\right| \int_{0}^{\infty} \frac{\mu_{1} \int_{0}^{\infty} a_{1}(t) G_{11}(t, s) \mathrm{d} A_{1}(t)}{\Gamma\left(\alpha_{1}\right)-\mu_{1} \int_{0}^{\infty} a_{1}(t) t^{\alpha_{1}-1} \mathrm{~d} A_{1}(t)} f_{1}(s, u(s), v(s)) \mathrm{d} s \\
& \leq \int_{0}^{\infty}\left|\frac{G_{11}\left(t_{2}, s\right)}{1+t_{2}^{\alpha_{1}-1}}-\frac{G_{11}\left(t_{1}, s\right)}{1+t_{2}^{\alpha_{1}-1}}\right| f_{1}(s, u(s), v(s)) \mathrm{d} s \\
& +\int_{0}^{\infty}\left|\frac{G_{11}\left(t_{1}, s\right)}{1+t_{2}^{\alpha_{1}-1}}-\frac{G_{11}\left(t_{1}, s\right)}{1+t_{1}^{\alpha_{1}-1}}\right| f_{1}(s, u(s), v(s)) \mathrm{d} s \\
& +\left|\frac{t_{2}^{\alpha_{1}-1}}{1+t_{2}^{\alpha_{1}-1}}-\frac{t_{1}^{\alpha_{1}-1}}{1+t_{1}^{\alpha_{1}-1}}\right| \int_{0}^{\infty} \frac{\mu_{1} \int_{0}^{\infty} a_{1}(t) G_{11}(t, s) \mathrm{d} A_{1}(t)}{\Gamma\left(\alpha_{1}\right)-\mu_{1} \int_{0}^{\infty} a_{1}(t) t^{\alpha_{1}-1} \mathrm{~d} A_{1}(t)} f_{1}(s, u(s), v(s)) \mathrm{d} s .
\end{aligned}
$$


Since

$$
\begin{aligned}
& \int_{0}^{\infty}\left|\frac{G_{11}\left(t_{2}, s\right)}{1+t_{2}^{\alpha_{1}-1}}-\frac{G_{11}\left(t_{1}, s\right)}{1+t_{2}^{\alpha_{1}-1}}\right| f_{1}(s, u(s), v(s)) \mathrm{d} s \\
& \leq \int_{0}^{t_{1}}\left|\frac{G_{11}\left(t_{2}, s\right)}{1+t_{2}^{\alpha_{1}-1}}-\frac{G_{11}\left(t_{1}, s\right)}{1+t_{2}^{\alpha_{1}-1}}\right| f_{1}(s, u(s), v(s)) \mathrm{d} s \\
& +\int_{t_{1}}^{t_{2}}\left|\frac{G_{11}\left(t_{2}, s\right)}{1+t_{2}^{\alpha_{1}-1}}-\frac{G_{11}\left(t_{1}, s\right)}{1+t_{2}^{\alpha_{1}-1}}\right| f_{1}(s, u(s), v(s)) \mathrm{d} s \\
& \quad+\int_{t_{2}}^{+\infty}\left|\frac{G_{11}\left(t_{2}, s\right)}{1+t_{2}^{\alpha_{1}-1}}-\frac{G_{11}\left(t_{1}, s\right)}{1+t_{2}^{\alpha_{1}-1}} f_{1}(s, u(s), v(s)) \mathrm{d} s\right| \\
& \leq \frac{1}{\Gamma\left(\alpha_{1}\right)} \int_{0}^{t_{1}} \frac{t_{2}^{\alpha_{1}-1}-t_{1}^{\alpha_{1}-1}|+|\left(t_{2}-s\right)^{\alpha_{1}-1}-\left(t_{1}-s\right)^{\alpha_{1}-1} \mid}{1+t_{2}^{\alpha_{1}-1}} f_{1}
\end{aligned}
$$$$
\cdot(s, u(s), v(s)) \mathrm{d} s
$$$$
+\frac{1}{\Gamma\left(\alpha_{1}\right)} \int_{t_{1}}^{t_{2}} \frac{\left|t_{2}^{\alpha_{1}-1}-t_{1}^{\alpha_{1}-1}\right|+\left|\left(t_{2}-s\right)^{\alpha_{1}-1}\right|}{1+t_{2}^{\alpha_{1}-1}} f_{1}(s, u
$$$$
\cdot(s), v(s)) \mathrm{d} s
$$$$
+\frac{1}{\Gamma\left(\alpha_{1}\right)} \int_{t_{2}}^{+\infty} \frac{\left|t_{2}^{\alpha_{1}-1}-t_{1}^{\alpha_{1}-1}\right|}{1+t_{2}^{\alpha_{1}-1}} f_{1}(s, u(s), v(s)) \mathrm{d} s
$$$$
\longrightarrow 0, \quad \text { as } t_{1} \longrightarrow t_{2} \text {. }
$$

In the same way, we can know

$\int_{0}^{\infty}\left|\frac{G_{11}\left(t_{1}, s\right)}{1+t_{2}^{\alpha_{1}-1}}-\frac{G_{11}\left(t_{1}, s\right)}{1+t_{1}^{\alpha_{1}-1}}\right| f_{1}(s, u(s), v(s)) \mathrm{d} s \longrightarrow 0, \quad$ as $t_{1} \longrightarrow t_{2}$.

So,

$\left|\frac{T_{1}(u, v)\left(t_{2}\right)}{1+t_{2}^{\alpha_{1}-1}}-\frac{T_{1}(u, v)\left(t_{1}\right)}{1+t_{1}^{\alpha_{1}-1}}\right| \longrightarrow 0, \quad$ as $t_{1} \longrightarrow t_{2}$.

Similar to (39)-(41), we have

$\left|\frac{T_{2}(u, v)\left(t_{2}\right)}{1+t_{2}^{\alpha_{2}-1}}-\frac{T_{2}(u, v)\left(t_{1}\right)}{1+t_{1}^{\alpha_{2}-1}}\right| \longrightarrow 0, \quad$ as $t_{1} \longrightarrow t_{2}$.

Therefore, $T(\Omega)$ is equicontinuous.

By $\left(\mathbf{H}_{1}\right)$ and (30), for any $\varepsilon>0$, there exists $\kappa>0$ such that

$$
\int_{\kappa}^{\infty} f\left(s, u_{n}(s), v_{n}(s)\right) \mathrm{d} s_{1}<\varepsilon .
$$

Due to $\lim _{t \longrightarrow+\infty}\left(t^{\alpha_{1}-1} / 1+t^{\alpha_{1}-1}\right)=1$, there exists sufficiently large $N_{1}>0$ such that, for any $t_{1}, t_{2}>N_{1}$, we have

$$
\left|\frac{t_{2}^{\alpha_{1}-1}}{1+t_{2}^{\alpha_{1}-1}}-\frac{t_{1}^{\alpha_{1}-1}}{1+t_{1}^{\alpha_{1}-1}}\right|<\varepsilon .
$$

Also because of $\lim _{t \rightarrow+\infty}\left((t-\kappa)^{\alpha_{1}-1} / 1+t^{\alpha_{1}-1}\right)=1$, there exists sufficiently large $N_{2}>\kappa$ such that, for any $t_{1}$, $t_{2}>N_{2}$ and $0 \leq s \leq \kappa$, we have

$$
\left|\frac{\left(t_{2}-s\right)^{\alpha_{1}-1}}{1+t_{2}^{\alpha_{1}-1}}-\frac{\left(t_{1}-s\right)^{\alpha_{1}-1}}{1+t_{1}^{\alpha_{1}-1}}\right|<\varepsilon .
$$

Choose $N>\max \left\{N_{1}, N_{2}\right\}$; for any $t_{1}, t_{2}>N$, we get

$$
\begin{aligned}
& \left|\frac{T_{1}(u, v)\left(t_{2}\right)}{1+t_{2}^{\alpha_{1}-1}}-\frac{T_{1}(u, v)\left(t_{1}\right)}{1+t_{1}^{\alpha_{1}-1}}\right| \\
& \quad \leq \int_{0}^{\infty}\left|\frac{G_{11}\left(t_{2}, s\right)}{1+t_{2}^{\alpha_{1}-1}}-\frac{G_{11}\left(t_{1}, s\right)}{1+t_{1}^{\alpha_{1}-1}}\right| f_{1}(s, u(s), v(s)) \mathrm{d} s \\
& \quad+\left|\frac{t_{2}^{\alpha_{1}-1}}{1+t_{2}^{\alpha_{1}-1}}-\frac{t_{1}^{\alpha_{1}-1}}{1+t_{1}^{\alpha_{1}-1}}\right| \int_{0}^{\infty} \frac{\mu_{1} \int_{0}^{\infty} a_{1}(t) G_{11}(t, s) \mathrm{d} A_{1}(t)}{\Gamma\left(\alpha_{1}\right)-\mu_{1} \int_{0}^{\infty} a_{1}(t) t^{\alpha_{1}-1} \mathrm{~d} A_{1}(t)} f_{1}(s, u(s), v(s)) \mathrm{d} s .
\end{aligned}
$$


In (47),

$$
\begin{aligned}
& \int_{0}^{\infty}\left|\frac{G_{11}\left(t_{2}, s\right)}{1+t_{2}^{\alpha_{1}-1}}-\frac{G_{11}\left(t_{1}, s\right)}{1+t_{1}^{\alpha_{1}-1}}\right| f_{1}(s, u(s), v(s)) \mathrm{d} s \\
& \leq \int_{0}^{t_{1}}\left|\frac{G_{11}\left(t_{2}, s\right)}{1+t_{2}^{\alpha_{1}-1}}-\frac{G_{11}\left(t_{1}, s\right)}{1+t_{1}^{\alpha_{1}-1}}\right| f_{1}(s, u(s), v(s)) \mathrm{d} s \\
& +\int_{t_{1}}^{t_{2}}\left|\frac{G_{11}\left(t_{2}, s\right)}{1+t_{2}^{\alpha_{1}-1}}-\frac{G_{11}\left(t_{1}, s\right)}{1+t_{1}^{\alpha_{1}-1}}\right| f_{1}(s, u(s), v(s)) \mathrm{d} s \\
& +\int_{t_{2}}^{\infty}\left|\frac{G_{11}\left(t_{2}, s\right)}{1+t_{2}^{\alpha_{1}-1}}-\frac{G_{11}\left(t_{1}, s\right)}{1+t_{1}^{\alpha_{1}-1}}\right| f_{1}(s, u(s), v(s)) \mathrm{d} s \\
& \leq \frac{1}{\Gamma\left(\alpha_{1}\right)} \int_{0}^{t_{1}}\left(\left|\frac{t_{2}^{\alpha_{1}-1}}{1+t_{2}^{\alpha_{1}-1}}-\frac{t_{1}^{\alpha_{1}-1}}{1+t_{1}^{\alpha_{1}-1}}\right|+\left|\frac{\left(t_{2}-s\right)^{\alpha_{1}-1}}{1+t_{2}^{\alpha_{1}-1}}-\frac{\left(t_{1}-s\right)^{\alpha_{1}-1}}{1+t_{1}^{\alpha_{1}-1}}\right|\right) f_{1}(s, u(s), v(s)) \mathrm{d} s \\
& +\frac{1}{\Gamma\left(\alpha_{1}\right)} \int_{t_{1}}^{t_{2}}\left(\left|\frac{t_{2}^{\alpha_{1}-1}}{1+t_{2}^{\alpha_{1}-1}}-\frac{t_{1}^{\alpha_{1}-1}}{1+t_{1}^{\alpha_{1}-1}}\right|+\left|\frac{\left(t_{2}-s\right)^{\alpha_{1}-1}}{1+t_{2}^{\alpha_{1}-1}}\right|\right) f_{1}(s, u(s), v(s)) \mathrm{d} s \\
& +\frac{1}{\Gamma\left(\alpha_{1}\right)} \int_{t_{2}}^{\infty}\left|\frac{t_{2}^{\alpha_{1}-1}}{1+t_{2}^{\alpha_{1}-1}}-\frac{t_{1}^{\alpha_{1}-1}}{1+t_{1}^{\alpha_{1}-1}}\right| f_{1}(s, u(s), v(s)) \mathrm{d} s \\
& \leq \frac{1}{\Gamma\left(\alpha_{1}\right)} \int_{0}^{\kappa}\left(\left|\frac{t_{2}^{\alpha_{1}-1}}{1+t_{2}^{\alpha_{1}-1}}-\frac{t_{1}^{\alpha_{1}-1}}{1+t_{1}^{\alpha_{1}-1}}\right|+\left|\frac{\left(t_{2}-s\right)^{\alpha_{1}-1}}{1+t_{2}^{\alpha_{1}-1}}-\frac{\left(t_{1}-s\right)^{\alpha_{1}-1}}{1+t_{1}^{\alpha_{1}-1}}\right|\right) f_{1}(s, u(s), v(s)) \mathrm{d} s \\
& +\frac{1}{\Gamma\left(\alpha_{1}\right)} \int_{\kappa}^{t_{1}}\left(\left|\frac{t_{2}^{\alpha_{1}-1}}{1+t_{2}^{\alpha_{1}-1}}-\frac{t_{1}^{\alpha_{1}-1}}{1+t_{1}^{\alpha_{1}-1}}\right|+\left|\frac{\left(t_{2}-s\right)^{\alpha_{1}-1}}{1+t_{2}^{\alpha_{1}-1}}-\frac{\left(t_{1}-s\right)^{\alpha_{1}-1}}{1+t_{1}^{\alpha_{1}-1}}\right|\right) f_{1}(s, u(s), v(s)) \mathrm{d} s \\
& +\frac{1}{\Gamma\left(\alpha_{1}\right)} \int_{\kappa}^{\infty}\left(\left|\frac{t_{2}^{\alpha_{1}-1}}{1+t_{2}^{\alpha_{1}-1}}-\frac{t_{1}^{\alpha_{1}-1}}{1+t_{1}^{\alpha_{1}-1}}\right|+1\right) f_{1}(s, u(s), v(s)) \mathrm{d} s \\
& +\frac{1}{\Gamma\left(\alpha_{1}\right)} \int_{\kappa}^{\infty}\left|\frac{t_{2}^{\alpha_{1}-1}}{1+t_{2}^{\alpha_{1}-1}}-\frac{t_{1}^{\alpha_{1}-1}}{1+t_{1}^{\alpha_{1}-1}}\right| f_{1}(s, u(s), v(s)) \mathrm{d} s \\
& \leq \frac{s \in[0, \kappa],(u, v) \in \Omega\left|f_{1}(s, u(s), v(s))\right|}{\Gamma\left(\alpha_{1}\right)} \int_{0}^{\kappa}\left(\left|\frac{t_{2}^{\alpha_{1}-1}}{1+t_{2}^{\alpha_{1}-1}}-\frac{t_{1}^{\alpha_{1}-1}}{1+t_{1}^{\alpha_{1}-1}}\right|+\left|\frac{\left(t_{2}-s\right)^{\alpha_{1}-1}}{1+t_{2}^{\alpha_{1}-1}}-\frac{\left(t_{1}-s\right)^{\alpha_{1}-1}}{1+t_{1}^{\alpha_{1}-1}}\right|\right) \mathrm{d} s \\
& +\frac{1}{\Gamma\left(\alpha_{1}\right)} \int_{\kappa}^{\infty}\left(\left|\frac{t_{2}^{\alpha_{1}-1}}{1+t_{2}^{\alpha_{1}-1}}-\frac{t_{1}^{\alpha_{1}-1}}{1+t_{1}^{\alpha_{1}-1}}\right|+\left|\frac{\left(t_{2}-s\right)^{\alpha_{1}-1}}{1+t_{2}^{\alpha_{1}-1}}-\frac{\left(t_{1}-s\right)^{\alpha_{1}-1}}{1+t_{1}^{\alpha_{1}-1}}\right|\right) f_{1}(s, u(s), v(s)) \mathrm{d} s \\
& +\frac{1}{\Gamma\left(\alpha_{1}\right)} \int_{\kappa}^{\infty}\left(\left|\frac{t_{2}^{\alpha_{1}-1}}{1+t_{2}^{\alpha_{1}-1}}-\frac{t_{1}^{\alpha_{1}-1}}{1+t_{1}^{\alpha_{1}-1}}\right|+1\right) f_{1}(s, u(s), v(s)) \mathrm{d} s \\
& +\frac{1}{\Gamma\left(\alpha_{1}\right)} \int_{\kappa}^{\infty}\left|\frac{t_{2}^{\alpha_{1}-1}}{1+t_{2}^{\alpha_{1}-1}}-\frac{t_{1}^{\alpha_{1}-1}}{1+t_{1}^{\alpha_{1}-1}}\right| f_{1}(s, u(s), v(s)) \mathrm{d} s \\
& \leq \frac{s \in[0, \kappa],(u, v) \in \Omega\left|f_{1}(s, u(s), v(s))\right|}{\Gamma\left(\alpha_{1}\right)} \int_{0}^{\kappa}\left(\left|\frac{t_{2}^{\alpha_{1}-1}}{1+t_{2}^{\alpha_{1}-1}}-\frac{t_{1}^{\alpha_{1}-1}}{1+t_{1}^{\alpha_{1}-1}}\right|+\left|\frac{\left(t_{2}-s\right)^{\alpha_{1}-1}}{1+t_{2}^{\alpha_{1}-1}}-\frac{\left(t_{1}-s\right)^{\alpha_{1}-1}}{1+t_{1}^{\alpha_{1}-1}}\right|\right) \mathrm{d} s \\
& +\frac{9}{\Gamma\left(\alpha_{1}\right)} \int_{\kappa}^{\infty} f_{1}(s, u(s), v(s)) \mathrm{d} s \\
& \leq \frac{s \in[0, \kappa],(u, v) \in \Omega\left|f_{1}(s, u(s), v(s))\right|}{\Gamma\left(\alpha_{1}\right)} 2 \kappa \varepsilon+\frac{9}{\Gamma\left(\alpha_{1}\right)} \varepsilon .
\end{aligned}
$$


By (47) and (48), we have

$$
\begin{aligned}
& \left|\frac{T_{1}(u, v)\left(t_{2}\right)}{1+t_{2}^{\alpha_{1}-1}}-\frac{T_{1}(u, v)\left(t_{1}\right)}{1+t_{1}^{\alpha_{1}-1}}\right| \\
& \leq \frac{\max _{s \in[0, \kappa],(u, v) \in \Omega}\left|f_{1}(s, u(s), v(s))\right|}{\Gamma\left(\alpha_{1}\right)} 2 \kappa \varepsilon+\frac{9}{\Gamma\left(\alpha_{1}\right)} \varepsilon \\
& \quad+\varepsilon \int_{0}^{\infty} \frac{\mu_{1} \int_{0}^{\infty} a_{1}(t) G_{11}(t, s) \mathrm{d} A_{1}(t)}{\Gamma\left(\alpha_{1}\right)-\mu_{1} \int_{0}^{\infty} a_{1}(t) t^{\alpha_{1}-1} \mathrm{~d} A_{1}(t)} f_{1}(s, u(s), v(s)) \mathrm{d} s .
\end{aligned}
$$

So, $T_{1}(u, v)$ is equicontinuous on $+\infty$, proof similar to (49), and we know $T_{2}(u, v)$ is equicontinuous on $+\infty$; thus, $T(u, v)$ is equicontinuous on $+\infty$. It follows from Lemma 4 that $T: X \longrightarrow X$ is relatively compact. Therefore, $T: X \longrightarrow X$ is completely continuous. The proof is completed.

Theorem 2. Assume that $\left(\boldsymbol{H}_{1}\right)$ holds; then, system (1) has at least one positive solution if $\omega\left(g_{1}^{*}+g_{2}^{*}+h_{1}^{*}+h_{2}^{*}\right)<1$.

Proof. Let

$$
\begin{gathered}
r \geq \frac{\omega\left(p_{1}^{*}+p_{2}^{*}\right)}{1-\omega\left(g_{1}^{*}+g_{2}^{*}+h_{1}^{*}+h_{2}^{*}\right)}, \\
K=\{(u, v) \in X,\|(u, v)\| \leq r\} .
\end{gathered}
$$

Now, we illustrate $T(K) \subset K$ for any $(u, v) \in K$ and $t \in[0$, $+\infty)$; by Lemma 3 and Remark 1, we have

$$
\begin{aligned}
\left|\frac{T_{1}(u, v)(t)}{1+t^{\alpha_{1}-1}}\right| & =\left|\int_{0}^{\infty} \frac{G_{1}(t, s)}{1+t^{\alpha_{1}-1}} f_{1}(s, u(s), v(s)) \mathrm{d} s\right| \\
& \leq \omega \int_{0}^{\infty}\left|f_{1}(s, u(s), v(s))\right| \mathrm{d} s \\
& \leq \omega\left(p_{1}^{*}+\left(g_{1}^{*}+h_{1}^{*}\right)\|(u, v)\|\right) \\
& \leq \omega\left(p_{1}^{*}+\left(g_{1}^{*}+h_{1}^{*}\right) r\right) \leq r .
\end{aligned}
$$

Similarly, we have

$$
\left|\frac{T_{2}(u, v)(t)}{1+t^{\alpha_{2}-1}}\right| \leq \omega\left(p_{2}^{*}+\left(g_{2}^{*}+h_{2}^{*}\right) r\right) \leq r .
$$

By (51) and (52),

$$
\begin{aligned}
\|T(u, v)\|= & \left\|T_{1}(u, v)\right\|+\left\|T_{2}(u, v)\right\| \leq \omega\left[\left(p_{1}^{*}+\left(g_{1}^{*}+h_{1}^{*}\right) r\right)\right. \\
& \left.+\left(p_{2}^{*}+\left(g_{2}^{*}+h_{2}^{*}\right) r\right)\right] \leq r .
\end{aligned}
$$

Therefore, $T(K) \subset K$. By Theorem 1, we know that $T$ : $K \longrightarrow K$ is completely continuous. So, by the Schauder fixed-point theorem, system (1) has at least one positive solution. The proof is completed.

Theorem 3. Assume that $\left(\boldsymbol{H}_{2}\right)$ holds; then, system (1) has a unique positive solution if $\omega\left(g_{1}^{*}+g_{2}^{*}+h_{1}^{*}+h_{2}^{*}\right)<1$.

Proof. From $\left(\mathbf{H}_{2}\right)$, we know

$$
\left|f_{i}(t, u, v)\right| \leq g_{i}(t)|u|+h_{i}(t)|v|+\left|f_{i}(t, 0,0)\right| .
$$

So, for any $(u, v) \in X$, we have

$$
\begin{aligned}
& \int_{0}^{\infty}\left|f_{i}(t, u(t), v(t))\right| \mathrm{d} t \\
& \quad \leq \int_{0}^{\infty}\left(\left(g_{i}(t)|u(t)|+h_{i}(t)|v(t)|+\left|f_{i}(t, 0,0)\right|\right)\right) \mathrm{d} t \\
& \quad=\int_{0}^{\infty}\left(\left(1+t^{\alpha_{1}-1}\right) g_{i}(t) \frac{|u(t)|}{1+t^{\alpha_{1}-1}}+\left(1+t^{\alpha_{2}-1}\right) h_{i}(t) \frac{|v(t)|}{1+t^{\alpha_{2}-1}}+\left|f_{i}(t, 0,0)\right|\right) \mathrm{d} t \\
& \quad \leq \int_{0}^{\infty}\left(\left(1+t^{\alpha_{1}-1}\right) g_{i}(t)\|u(t)\|+\left(1+t^{\alpha_{2}-1}\right) h_{i}(t)\|v(t)\|+\left|f_{i}(t, 0,0)\right|\right) \mathrm{d} t \\
& \quad=\int_{0}^{\infty}\left(1+t^{\alpha_{1}-1}\right) g_{i}(t) \mathrm{d} t\|u(t)\|+\int_{0}^{\infty}\left(1+t^{\alpha_{2}-1}\right) h_{i}(t) \mathrm{d} t\|v(t)\|+\int_{0}^{\infty}\left|f_{i}(t, 0,0)\right| \mathrm{d} t \\
& \quad=g_{i}^{*}\|u\|+h_{i}^{*}\|v\|+\int_{0}^{\infty}\left|f_{i}(t, 0,0)\right| \mathrm{d} t \leq\left(g_{i}^{*}+h_{i}^{*}\right)\|(u, v)\|+\int_{0}^{\infty}\left|f_{i}(t, 0,0)\right| \mathrm{d} t .
\end{aligned}
$$


For any $\left(u_{1}, v_{1}\right),\left(u_{2}, v_{2}\right) \in X$ and $t \in[0,+\infty)$, by Lemma 3 , we have

$$
\begin{aligned}
& \left|\frac{T_{1}\left(u_{1}, v_{1}\right)(t)-T_{1}\left(u_{2}, v_{2}\right)(t)}{1+t^{\alpha_{1}-1}}\right| \\
& =\left|\int_{0}^{\infty} \frac{G_{1}(t, s)}{1+t^{\alpha_{1}-1}}\right| f_{1}\left(s, u_{1}(s), v_{1}(s)\right)-f_{1}\left(s, u_{2}(s), v_{2}(s)\right)|\mathrm{d} s| \\
& \leq \omega \int_{0}^{\infty}\left(g_{1}(s)\left|\left(u_{1}(s)-u_{2}(s)\right)\right|+h_{1}(s)\left|\left(v_{1}(s)-v_{2}(s)\right)\right|\right) \mathrm{d} s \\
& \leq \omega \int_{0}^{\infty}\left(\left(1+s^{\alpha_{1}-1}\right) g_{1}(s) \frac{\left|\left(u_{1}(s)-u_{2}(s)\right)\right|}{1+s^{\alpha_{1}-1}}+\left(1+s^{\alpha_{1}-1}\right) h_{1}\right. \\
& \left.\quad(s) \frac{\left|\left(v_{1}(s)-v_{2}(s)\right)\right|}{1+s^{\alpha_{1}-1}}\right) \mathrm{d} s \\
& \leq \omega\left(g_{1}^{*}\left\|u_{1}-u_{2}\right\|+h_{1}^{*}\left\|v_{1}-v_{2}\right\|\right) .
\end{aligned}
$$

By the similar proof, we have

$\left|\frac{T_{2}\left(u_{1}, v_{1}\right)(t)-T_{2}\left(u_{2}, v_{2}\right)(t)}{1+t^{\alpha_{2}-1}}\right| \leq \omega\left(g_{2}^{*}\left\|u_{1}-u_{2}\right\|+h_{2}^{*}\left\|v_{1}-v_{2}\right\|\right)$.

Now, inequalities (56) and (57) can show that

$$
\|T(u, v)\| \leq \omega\left(g_{1}^{*}+g_{2}^{*}+h_{1}^{*}+h_{2}^{*}\right)\left(\left\|u_{1}-u_{2}\right\|+\left\|v_{1}-v_{2}\right\|\right) .
$$

Thus, by the Banach contraction mapping theorem that $T$ has a unique fixed point in $X$, system (1) has a unique positive solution. The proof is completed.

\section{An Example}

Consider the following fractional differential system:

$$
\left\{\begin{array}{l}
D_{0+}^{(5 / 2)} u(t)+f_{1}(t, u(t), v(t))=0, \\
D_{0+}^{(7 / 2)} v(t)+f_{2}(t, u(t), v(t))=0, \quad 0<t<+\infty \\
u(0)=u^{\prime}(0)=0 \\
D_{0+}^{(3 / 2)} u(+\infty)=\frac{1}{5} \int_{0}^{\infty} e^{-t} u(t) \mathrm{d} t \\
v(0)=v^{\prime}(0)=v^{\prime \prime}(0)=0 \\
D_{0+}^{(5 / 2)} v(+\infty)=\frac{1}{4} \int_{0}^{\infty} e^{-t} v(t) \mathrm{d} t
\end{array}\right.
$$

where $\alpha_{1}=(5 / 2), \alpha_{2}=(7 / 2), \quad \mu_{1}=(1 / 5), \mu_{2}=(1 / 4), A_{1}$ $(t)=A_{2}(t)=t, a_{1}(t)=e^{-t}=a_{2}(t)=e^{-t}$. Then, we have

$$
\begin{aligned}
\int_{0}^{\infty} a_{1}(t) \mathrm{d} A_{1}(t) & =\int_{0}^{\infty} a_{2}(t) \mathrm{d} A_{2}(t)=\int_{0}^{\infty} e^{-t} \mathrm{~d} t=1<+\infty, \\
\int_{0}^{\infty} a_{1}(t) t^{\alpha_{1}-1} \mathrm{~d} A_{1}(t) & =\int_{0}^{\infty} e^{-t} t^{(3 / 2)} \mathrm{d} t=2.3562<+\infty \\
\int_{0}^{\infty} a_{2}(t) t^{\alpha_{2}-1} \mathrm{~d} A_{2}(t) & =\int_{0}^{\infty} e^{-t} t^{(5 / 2)} \mathrm{d} t=3.3233<+\infty \\
\omega & =2.0929 . \\
\operatorname{Take} & \\
f_{1}(t, u(t), v(t)) & =\frac{1}{1+e^{t}}+\frac{u(t)|\cos t|}{\left(1+t^{(3 / 2)}\right) e^{7 t}}+\frac{v(t)|\sin t|}{7\left(1+t^{(5 / 2)}\right) e^{3 t}}, \\
f_{2}(t, u(t), v(t)) & =\frac{1}{2\left(1+e^{t}\right)}+\frac{u(t)}{\left(1+t^{(3 / 2)}\right) e^{6 t}}+\frac{v(t)}{3\left(1+t^{(5 / 2)}\right) e^{4 t}} .
\end{aligned}
$$

Let

$$
\begin{aligned}
& g_{1}(t)=\frac{1}{\left(1+t^{(3 / 2)}\right) e^{7 t}}, \\
& g_{2}(t)=\frac{1}{\left(1+t^{(3 / 2)}\right) e^{6 t}}, \\
& h_{1}(t)=\frac{1}{7\left(1+t^{(5 / 2)}\right) e^{3 t}}, \\
& h_{2}(t)=\frac{1}{3\left(1+t^{(5 / 2)}\right) e^{4 t}} .
\end{aligned}
$$

Through calculation, we get

$$
\begin{aligned}
& g_{1}^{*}= \int_{0}^{\infty}\left(1+t^{\alpha_{1}-1}\right) g_{1}(t) \mathrm{d} t=\int_{0}^{\infty} e^{7 t} \mathrm{~d} t=\frac{1}{7}, \\
& g_{2}^{*}=\int_{0}^{\infty}\left(1+t^{\alpha_{1}-1}\right) g_{2}(t) \mathrm{d} t=\int_{0}^{\infty} e^{6 t} \mathrm{~d} t=\frac{1}{6}, \\
& h_{1}^{*}=\int_{0}^{\infty}\left(1+t^{\alpha_{2}-1}\right) h_{1}(t) \mathrm{d} t=\frac{1}{7} \int_{0}^{\infty} e^{3 t} \mathrm{~d} t=\frac{1}{21}, \\
& h_{2}^{*}=\int_{0}^{\infty}\left(1+t^{\alpha_{2}-1}\right) h_{2}(t) \mathrm{d} t=\frac{1}{3} \int_{0}^{\infty} e^{4 t} \mathrm{~d} t=\frac{1}{12}, \\
& \omega\left(g_{1}^{*}+g_{2}^{*}+h_{1}^{*}+h_{2}^{*}\right)=2.0929\left(\frac{1}{7}+\frac{1}{6}+\frac{1}{21}+\frac{1}{12}\right) \\
&= 0.9219<1 .
\end{aligned}
$$

Then, by Theorem 2, system (59) has at least one positive solution.

\section{Data Availability}

No data were used to support this study.

\section{Conflicts of Interest}

The authors declare that they have no conflicts of interest. 


\section{Acknowledgments}

This work was supported by the National Natural Science Foundation of China (11701252 and 61703194), the Science Research Foundation for Doctoral Authorities of Linyi University (LYDX2016BS080), the Natural Science Foundation of Shandong Province of China (ZR2018MA016), and the Applied Mathematics Enhancement Program of Linyi University.

\section{References}

[1] R. V. Thomann and J. A. Mueller, Principles of Surface Water Quality Modeling and Control, New York Harper Row, New York, NY, USA, 1987.

[2] S. Westerlund, "Dead matter has memory!" Physica Scripta, vol. 43, no. 2, pp. 174-179, 1991.

[3] X. Zhang, L. Liu, Y. Wu, and Y. Cui, "A sufficient and necessary condition of existence of blow-up radial solutions for a $k$-Hessian equation with a nonlinear operator," Nonlinear Analysis: Modelling and Control, vol. 25, pp. 126-143, 2020.

[4] C. Chen, K. Li, Y. Chen, and Y. Huang, "Two-grid finite element methods combined with Crank-Nicolson scheme for nonlinear Sobolev equations," Advances in Computational Mathematics, vol. 45, no. 2, pp. 611-630, 2019.

[5] X. Zhang, L. Liu, Y. Wu, and Y. Cui, "The existence and nonexistence of entire large solutions for a quasilinear Schrödinger elliptic system by dual approach," Journal of Mathematical Analysis and Applications, vol. 464, no. 2, pp. 1089-1106, 2018.

[6] X. Zhang, J. Jiang, Y. Wu, and Y. Cui, "Existence and asymptotic properties of solutions for a nonlinear Schrödinger elliptic equation from geophysical fluid flows," Applied Mathematics Letters, vol. 90, pp. 229-237, 2019.

[7] X. Zhao and W. Ge, "Unbounded solutions for a fractional boundary value problems on the infinite interval," Acta Applicandae Mathematicae, vol. 109, no. 2, pp. 495-505, 2010.

[8] A. Arara, M. Benchohra, N. Hamidi, and J. J. Nieto, "Fractional order differential equations on an unbounded domain," Nonlinear Analysis: Theory, Methods \& Applications, vol. 72, no. 2, pp. 580-586, 2010.

[9] R. P. Agarwal, M. Benchohra, S. Hamani, and S. Pinelas, "Boundary value problems for differential equations involving RiemannCLiouville fractional derivative on the half-line," Dynamics of Continuous, Discrete and Impulsive Systems Series A: Mathematical Analysis, vol. 18, no. 2, pp. 235-244, 2011.

[10] W. Jiang, "Solvability for fractional differential equations at resonance on the half line," Applied Mathematics and Computation, vol. 247, pp. 90-99, 2014.

[11] G. Wang, "Explicit iteration and unbounded solutions for fractional integral boundary value problem on an infinite interval," Applied Mathematics Letters, vol. 47, pp. 1-7, 2015.

[12] M. Jia, H. Zhang, and Q. Chen, "Existence of positive solutions for fractional differential equation with integral boundary conditions on the half-line," Boundary Value Problems, vol. 2016, p. 104, 2016.

[13] F. Toumi and Z. Zine El Abidine, "Existence of multiple positive solutions for nonlinear fractional boundary value problems on the half-line," Mediterranean Journal of Mathematics, vol. 13, no. 5, pp. 2353-2364, 2016.
[14] L. Zhang, B. Ahmad, and G. Wang, "Monotone iterative method for a class of nonlinear fractional differential equations on unbounded domains in Banach spaces," Filomat, vol. 31, no. 5, pp. 1331-1338, 2017.

[15] X. Zhang, L. Liu, Y. Wu, and Y. Cui, "Existence of infinitely solutions for a modified nonlinear Schrödinger eqaution via dual approach," Electronic Journal of Differential Equations, vol. 2147, pp. 1-15, 2018.

[16] S. Y. Li and C. B. Zhai, "Positive solutions for a new class of Hadamard fractional differential equations on infinite intervals," Journal of Inequalities and Applications, vol. 2019, p. 150, 2019.

[17] S. H. Liang and J. H. Zhang, "Existence of three positive solutions of $m$-point boundary value problems for some nonlinear fractional differential equations on an infinite interval," Computers \& Mathematics with Applications, vol. 61, no. 11, pp. 3343-3354, 2011.

[18] K. S. Miller and B. Ross, An Introduction to the Fractional Calculus and Fractional Differential Equations, John Wiley, New York, NY, USA, 1993.

[19] I. Podlubny, "Fractional differential equations," Mathematics in Science and Engineering, Vol. 198, Academic Press, New York, NY, USA, 1999.

[20] Y. S. Liu, "Boundary value problem for second order differential equations on unbounded domain," Acta Applicandae Mathematicae, vol. 4, no. 3, pp. 211-216, 2002.

[21] C. Corduneanu, Integral Equations and Stability of Feedback Systems, Academic Press, New York, NY, USA, 1973. 